\title{
A possible way to relate the "covariantization" and the negative dimensional integration method in the light-cone gauge
}

\author{
Alfredo T. Suzuki and Ricardo Bentín f \\ Instituto de Física Teórica - UNESP. \\ Rua Pamplona 145, CEP 01405-900, SP, Brasil.
}

\begin{abstract}
In this work we present a possible way to relate the method of covariantizing the gauge dependent pole and the negative dimensional integration method for computing Feynman integrals pertinent to the light-cone gauge fields. Both techniques are applicable to the algebraic light-cone gauge and dispense with prescriptions to treat the characteristic poles.
\end{abstract}

PACS: 02.90.+p; 12.38.Bx

${ }^{a}$ e-mail: suzuki@ift.unesp.br

${ }^{z}$ e-mail: rbentin@ift.unesp.br 


\section{Introduction.}

Recently, Suzuki and Schmidt [1] have calculated several one- and two-loop Feynman integrals in the algebraic type gauges such as the light-cone and the Coulomb ones using the negative dimensional integration (NDIM) approach. For the former gauge, they have proven that the NDIM approach can dispense with prescriptions and partial fractionings as well as avoiding parametric integrals and integrations over components. The only requirement in NDIM is the correct choice of the light-like basis vectors that generate the four-dimensional space-time. In this line, the second light-like "dual" vector $m^{\mu},\left(m^{2}=0\right)$, plays a key role in the whole process, otherwise the calculations go wrong, violating causality and so on. Actually, the gauge proper is defined with the one light-like vector, usually denoted by $n^{\mu},\left(n^{2}=0\right)$, such that $n \cdot A=0$, where $A_{\mu}$ is the gauge field; however, this condition is not enough. A residual gauge freedom remains that must be eliminated via $m^{\mu}$ constraint.

The NDIM technique totally circumvents the need for prescriptions, since the evaluation is done in negative dimension, or as understood, as fermionic integrals (polynomial integrands) in positive dimension. Here we would like to emphasize that the prescriptionlessness of the NDIM approach in the light-cone gauge has nothing whatsoever to do with the residual gauge freedom mentioned above. It has all to do with the polynomial character of the fermionic integrands.

On the other hand, there is a technique coined by Suzuki [2] as covarianti- 
zation to treat light-cone integrals. This technique is causal and reproduces the results obtained through the use of the Mandelstam-Leibbrandt prescription [3]. This fact remains almost forgotten, even though the covariantization manifestly guarantees the absence of zero mode frequencies that spoil causality. Of course, here the technique uses parametric integrations and integration over components, and all the technology of complex analysis to treat the singularities in a proper way. The thrust of the technique lies in that it does not require a prescription for the light-cone pole; it "converts" this pole in a "covariant" pole whose treatment is well-grounded and established since the early days of quantum field theory. The burden of this technique and its most severe drawback is that it requires an additional parametric integration to be performed, a task which can be very demanding.

Since we are going to use the light-cone gauge throughout this work, we will briefly describe the light-cone coordinates. Using the metric $(+,-,-,-)$, then a general contravariant four-vector is given by:

$$
x^{\mu}=\left(x^{+}, x^{-}, x^{i}\right), i=1,2,
$$

where:

$$
\begin{aligned}
x^{ \pm} & =\frac{1}{\sqrt{2}}\left(x^{0} \pm x^{3}\right), \\
& =\frac{1}{\sqrt{2}}\left(x_{0} \mp x_{3}\right)=x_{\mp}, \\
x^{i} & \equiv(\hat{x})^{i}=\left(x^{1}, x^{2}\right)
\end{aligned}
$$


Now, defining the light-like four-vectors:

$$
\begin{aligned}
n_{\mu} & =\frac{1}{\sqrt{2}}(1,0,0,1), \\
m_{\mu} & =\frac{1}{\sqrt{2}}(1,0,0,-1),
\end{aligned}
$$

we observe that

$$
\begin{aligned}
& x^{+}=x^{\mu} n_{\mu}, \\
& x^{-}=x^{\mu} m_{\mu},
\end{aligned}
$$

and the scalar product becomes:

$$
x^{\mu} y_{\mu}=x^{+} y^{-}+x^{-} y^{+}-\hat{x} \hat{y},
$$

i.e.,

$$
x^{2}=2 x^{+} x^{-}-\hat{x}^{2}
$$

This means that the metric is now:

$$
g^{\mu \nu}=\left(\begin{array}{llll}
+ & - & 1 & 2 \\
0 & 1 & 0 & 0 \\
1 & 0 & 0 & 0 \\
0 & 0 & 1 & 0 \\
0 & 0 & 0 & 1
\end{array}\right) \quad \begin{aligned}
& + \\
& - \\
& 1 \\
& 2
\end{aligned}
$$

\section{What is meant by covariantization}

Here is a brief review of the "covariantization" technique which was proposed by Suzuki [2]. The idea is quite simple. In light-cone coordinates, the square of a four-momentum is:

$$
q^{2}=2 q^{+} q^{-}-\hat{q}^{2}
$$


As long as $q^{-} \neq 0$, we can write $q^{+}$as

$$
q^{+}=\frac{q^{2}+\hat{q}^{2}}{2 q^{-}}
$$

We note that this dispersion relation almost guarantees that real gauge fields for which $q^{2}=0$ (real photons or real gluons for example) are transverse; the residual gauge freedom, that is left to be dealt with so that fields be manifestly transverse comes from the presence of the $q^{-}$in the denominator of the expression above.

This implies that in the light-cone gauge the characteristic pole becomes

$$
\frac{1}{q^{+}}=\frac{2 q^{-}}{q^{2}+\hat{q}^{2}}
$$

The important thing here is that the condition $q^{-} \neq 0$ warranties the causal structure of this technique since it eliminates the troublesome $q^{-}=0$ modes. Elimination of these modes restores the physically acceptable results as can manifestly be seen in the causal prescription [4] for the light-cone gauge.

\section{$3 \quad$ Negative dimension integration.}

This method of integrating Feynman loop integrals is a follow up development of the early attempts by Halliday, and Ricotta [5] in this direction during the mid 80 's. In the late 90 's it was tested in the case of working in the light-cone gauge [6]. The best way to illustrate this method is through an example. So we will use the computation of the following integral:

$$
I_{i j k}=\int d^{2 \omega} q \cdot(q-a)^{2 i}(q-b)^{2 j}\left(q^{+}\right)^{k}
$$


Note that for $i=j=k=-1$ this integral is the basic one-loop integral in the light-cone gauge. In NDIM, we start with this integral considering positive values for the exponents $i, j$, and $k$ and negative dimension $D=2 \omega$ (or as state above, as a fermionic polynomial integration in positive dimension). First of all, let us define the generating Gaussian functional for the integral in question, that is,

$$
I_{\text {Gauss }}=\int d^{2 \omega} q \cdot e^{-\alpha(q-a)^{2}-\beta(q-b)^{2}-\gamma q^{+}}
$$

which clearly can be rewritten as

$$
I_{\text {Gauss }}=\sum_{i j k=0}^{\infty}(-)^{i+j+k} \frac{\alpha^{i}+\beta^{j}+\gamma^{k}}{i ! j ! k !} \overbrace{\int d^{2 \omega} q \cdot(q-a)^{2 i}(q-b)^{2 j}\left(q^{+}\right)}^{I_{i j k}} .
$$

On the other hand, performing the momentum integration, which is not difficult to do since it is a Gaussian integral, we arrive at

$$
I_{\text {Gauss }}=\left(\frac{\pi}{\alpha+\beta}\right)^{\omega} e^{-\frac{1}{\alpha+\beta}\left[\alpha \beta(a-b)^{2}-\alpha \gamma a^{+} \beta \gamma b^{+}\right]} .
$$

Expanding the last exponential and also using the multinomial expansion, we obtain

$$
I_{\text {Gauss }}=\pi^{\omega} \sum_{n_{i}=0}^{\infty}(-)^{n_{123}} \frac{\alpha^{n_{124}} \beta^{n_{135}} \gamma^{n_{23}}}{n_{1} ! n_{2} ! n_{3} ! n_{4} ! n_{5} !}\left(-n_{123}-\omega\right) !(a-b)^{n_{1}}\left(a^{+}\right)^{n_{2}}\left(b^{+}\right)^{n_{3}},
$$

where $i=1 \cdots 5, n_{a b c}=n_{a}+n_{b}+n_{c}$. Comparing now Eq. (3) and Eq. (雨) we conclude that $I_{i j k}$ is given by:

$$
\begin{aligned}
I_{i j k}= & \pi^{\omega}(-)^{i+j+k} \Gamma(1+i) \Gamma(1+j) \Gamma(1+k) \sum_{n_{i}=0}^{\infty}(-)^{n_{123}} \frac{\alpha^{n_{124}} \beta^{n_{135}} \gamma^{n_{23}}}{n_{1} ! n_{2} ! n_{3} ! n_{4} ! n_{5} !}\left(-n_{123}-\omega\right) ! \\
& \cdot(a-b)^{n_{1}}\left(a^{+}\right)^{n_{2}}\left(b^{+}\right)^{n_{3}} \cdot \delta_{n_{124}, i} \delta_{n_{135}, j} \delta_{n_{23}, k} \delta_{n_{45},-n_{123}-\omega} .
\end{aligned}
$$


The $\delta$ 's form a system of equations which in this case have four constraints,

$$
\begin{aligned}
n_{1}+n_{2}+n_{4} & =i, \\
n_{1}+n_{3}+n_{5} & =j, \\
n_{2}+n_{3} & =k, \\
n_{1}+n_{2}+n_{3}+n_{4}+n_{5}+\omega & =0 .
\end{aligned}
$$

Clearly the usual matrix equation does not close and no solution can be found since there are five unknowns and only four equations. The system can only be solved when we pretend one of the unknows is a known variable. Of course, there is no preferred unknown to play this role; we can choose it in several ways. In fact, in the present case, there are $C_{4}^{5}$ ways to do this, i.e., five solutions. One such solution is a trivial one, and then we have two sets of pairs of solutions (each pair belonging to a peculiar region of external momenta). And the general solution for the pertinent Feynman integral is constructed as a linear combination of the particular solutions we have for the system, according to the outcoming variables defined by the external momenta.

Each general solution will have as a rule two factors: a coefficient factor of gamma functions which we express in terms of Pochhammer's symbols and eventually multi-indexed series which is typically generalized hypergeometric functions of some sort, whose parameters depends on the exponents of propagators and dimension as well as the external momenta and vectors $n$ and $m$. The next 
step is to perform an analytic continuation of exponents into the negative values and positive dimension via a property of Pochhammer's symbols, such that we obtain for our present example,

$$
\begin{aligned}
I_{i j k}= & \pi^{\omega}\left[(a-b)^{2}\right]^{\rho-k}\left\{\left(b^{+}\right)^{k} A_{2} F_{1}(-k, \omega+j ; 1+j-\rho \mid z)\right\} \\
& \left.+\left(a^{+}\right)^{\rho-j}\left(b^{+}\right)^{-i-\omega} C_{2} F_{1}(\omega+i, \rho+\omega ; 1+\rho-j \mid z)\right\}
\end{aligned}
$$

where

$$
\begin{aligned}
& A=(-j \mid \rho)(-i \mid i+j+\omega)(\rho+\omega \mid-2 \rho-\omega+k), \\
& C=(-j \mid i+j+\omega)(-i \mid i+k-\rho)(-k \mid j+k-\rho),
\end{aligned}
$$

with $z=\frac{a \cdot n}{b \cdot n}$, and $\rho=i+j+k+\omega$. Also, the Pochhammer symbols is defined as:

$$
(a \mid b)=(a)_{b}=\frac{\Gamma(a+b)}{\Gamma(a)}
$$

with the property: $(a \mid b+c)=(a+b \mid c)(a \mid b)$. Taking the limit $i, j, k \rightarrow-1$ (i.e. positive dimension now) and using a property of the hypergeometric functions, it is possible to write it as just one term

$$
I_{-1-1-1}=\pi^{\omega} \frac{\left[(a-b)^{2}\right]^{\omega-2}}{a^{+}} \Gamma(2-\omega) B(\omega-1, \omega-1){ }_{2} F_{1}(1, \omega-1 ; 2 \omega-2 \mid u)
$$

Now, if we also make the limit $a \rightarrow 0$, the result is in complete agreement with that one found in Ref.[0], but it is a result in the context of the PV prescription for the light-cone gauge, which is wrong. We use the above computation in order to show how the negative integration method works. Now we are going to obtain the right answer as it was done in Ref. [6]. To do this, first note that when we 
defined the light-cone coordinates in Eq.11, there exists the dual of $n_{\mu}$, which we called $m_{\mu}$, and in light-cone computations, this vector always appears in the numerator of the integrands. Keeping this in mind, we will compute the following integral using NDIM technology:

$$
T_{i j k}=\int d^{2 \omega} q \cdot\left[(q-p)^{2}\right]^{i}\left(q^{+}\right)^{j}\left(q^{-}\right)^{k}, \quad k \geq 0 .
$$

Using the same procedure as above, we get

$$
T_{i j k}=(-\pi)^{\omega}\left(\frac{-2 p^{+} p^{-}}{n \cdot m}\right)^{i+\omega}\left(p^{+}\right)^{j}\left(p^{-}\right)^{k} \frac{(1-i-\omega \mid 2 i+\omega)}{(1+j \mid i+\omega)(1+k \mid i+\omega)}
$$

Note that since from the start we must have $k \geq 0$ the Pochhammer symbol containing the exponent $k$, namely, $(1+k \mid i+\omega)$ cannot be analytic continued and the final result, after analytic continuation to positive dimension and negative values of exponents $i$ and $j$ is

$$
T_{i j k}=\pi^{\omega}\left(\frac{2 p^{+} p^{-}}{n \cdot m}\right)^{i+\omega}\left(p^{+}\right)^{j}\left(p^{-}\right)^{k} \frac{(-j \mid-i-\omega)}{(i+\omega \mid-2 i-\omega)(1+k \mid i+\omega)}
$$

This result is in complete agreement with those obtained via causal prescriptions, such as the Mandelstam-Leibbrandt, but in this case without the use of any prescriptions.

\section{How they are related.}

Negative dimensional integration method is a prescriptionless method in the lightcone gauge. So it is the "covariantization" technique. Then, the natural question to ask is whether they are related to each other in any way. Let us consider for 
definiteness and simplicity but without loss of generality, the general form of the one-loop light-cone integral,

$$
F(p)=\int d^{2 \omega} q \cdot \frac{f(q, p, n)}{g(q, p)\left(q^{+}\right)^{\gamma}}, \quad \gamma>0
$$

where $f(q, p, n)$ and $g(q, p)$ are some well behaved functions. Also, let $\gamma=\alpha+\beta$, with $\alpha$ and $\beta$ being non-negative numbers. This means that we have two sets of choices for $\alpha$ and $\beta$. The first one is $\alpha \geq 0$ and $\beta>0$. The second one is $\alpha>0$ and $\beta \geq 0$. Then Eq.(6) will be,

$$
F(p)=\int d^{2 \omega} q \cdot \frac{f(q, p, n)}{g(q, p)\left(q^{+}\right)^{\alpha}\left(q^{+}\right)^{\beta}}
$$

In our analysis, we can choose whatever case. It is indifferent which one we take, since actually we can interchange $\alpha \leftrightarrow \beta$ and the conclusion does not change. Therefore we take $\alpha>0$ and $\beta \geq 0$ for definiteness. Use of the "covariantization" method means that for the $\beta$-pole we have,

$$
\frac{1}{\left(q^{+}\right)^{\beta}}=\frac{2^{\beta}\left(q^{-}\right)^{\beta}}{\left(q^{2}+\hat{q}^{2}\right)^{\beta}}
$$

Since we have chosen the case where $\alpha$ is strictly positive, $\alpha>0$, we keep this $\alpha$-pole as the caracteristic light-cone pole, and then

$$
F(p)=\int d^{2 \omega} q \cdot \frac{f(q, p, n) 2^{\beta}\left(q^{-}\right)^{\beta}}{g(q, p)\left(q^{2}+\hat{q}^{2}\right)^{\beta}\left(q^{+}\right)^{\alpha}}=\int d^{2 \omega} q \cdot \frac{F(q, p, n)\left(q^{-}\right)^{\beta}}{G(q, p)\left(q^{+}\right)^{\alpha}}
$$

where we have defined $F(q, p, n)=2^{\beta} f(q, p, n)$ and $G(q, p)=g(q, p)\left(q^{2}+\hat{q}^{2}\right)^{\beta}$. Since $\beta \geq 0$ and $\alpha>0$, we can see that this is the very condition we used to define the prescriptionless NDIM integral in negative dimensions. To illustrate 
these manipulations, take the following case: $f(q, p, n)=2^{\beta}\left(q^{2}-\hat{q}^{2}\right)^{-\beta}$ and $g(q, p)=(q-p)^{2}$, so that Eq.(7) takes the form:

$$
F(p)=\int d^{2 \omega} q \cdot \frac{\left(q^{-}\right)^{\beta}}{(q-p)^{2}\left(q^{+}\right)^{\alpha}}
$$

which is nothing more than the starting integral $T_{i j k}$ for the negative dimensional integration method. Remember that after the analytic continuation was made, we had to take the values of $\alpha=-1$ and $\beta=0$ and put it in Eq.(5). On the other hand, if we use the identities of the "covariantization" technique, the integral of Eq.(17) we arrive is,

$$
F(p)=\int d^{2 \omega} q \cdot \frac{1}{(q-p)^{2}\left(q^{+}\right)^{\alpha+\beta}}
$$

but $\alpha+\beta=1$ and with these values the above integral yields (using the covariantization) [2]

$$
F(p)=i(-\pi)^{\omega}\left(2 p^{+} p^{-}\right)^{\omega-1} \frac{\Gamma(2-\omega) \Gamma(\omega-1)}{\Gamma(\omega)},
$$

If we manipulate the result obtained in Eq.(5) (computed using negative integration) and compare with the above Eq.(9) (computed using "covariantization"), we will find that they are the same solution.

\section{Conclusions.}

Here we have shown that the two prescriptionless ways to treat the spurious poles of the light-cone gauge, are in fact related to each other. The results in both cases are in concordance with those ones in which some causal prescription is used. It is quite clear from the start that the covariantization method is done 
in positive dimensions whereas the NDIM is done in negative dimensions. What we were interested in was to see the possibility of relating one with the other and find some common grounds for both techniques. What we have found is that the covariantization technique, which ensures the troublesome $q^{-}=0$ modes to be left out of the computation in a natural way can be seen as the crucial factor from which to start with in negative dimensions. In other words, since $q^{-} \neq 0$ throughtout any computation to be done, one sees that the four-vector $m^{\mu}$ cannot be left out of the general structure in NDIM applied to the light-cone gauge.

\section{Acknowledgments:}

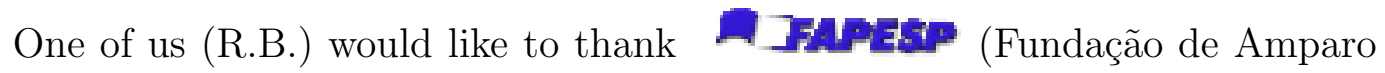
à Pesquisa do Estado de São Paulo) for financial support.

\section{References}

[1] A. Suzuki, A.G.M. Schmidt, Eur. Phys. J. C 5 (1998) 175.

A. Suzuki, A.G.M. Schmidt, Can. J. Phys. 78 (2000) 769.

A. Suzuki, A.G.M. Schmidt, J. Phys. A 33 (2000) 3713.

A. Suzuki, A.G.M. Schmidt, Phys. Lett. B 494 (2000) 332.

[2] A.T. Suzuki, Mod. Phys. Lett. A 825 (1993) 2365.

[3] S. Mandelstam, Nucl. Phys. B 213 (1983) 149.

G. Leibbrantd, Phys. Rev. D 29 (1984) 1699.

[4] B.M. Pimentel and A.T. Suzuki, Phys. Rev. D 42 (1990) 2115. 
[5] I.G. Halliday and R. Ricotta, Phys. Lett. B 193 (1987) 241.

G.V. Dunne and I.G. Halliday, Phys Lett. B 193 (1987) 247.

[6] A. Suzuki, A. Schmidt, R Bentín, Nucl. Phys. B 537 (1999) 549.

[7] D.M. Capper, J.J. Dulwichand M.J. Litvak, Nucl. Phys. B 241 (1984) 463. 